\title{
Aa. Vv., Adverbiaux et topiques
}

\section{Chiara Preite}

\section{OpenEdition}

\section{Journals}

\section{Édition électronique}

URL : http://journals.openedition.org/studifrancesi/35431

DOI : 10.4000/studifrancesi.35431

ISSN : 2427-5856

\section{Éditeur}

Rosenberg \& Sellier

\section{Édition imprimée}

Date de publication : 1 novembre 2005

Pagination : 468-469

ISSN : 0039-2944

\section{Référence électronique}

Chiara Preite, «Aa. VV., Adverbiaux et topiques », Studi Francesi [En ligne], 146 (XLIX | II) | 2005, mis en ligne le 30 novembre 2015, consulté le 18 avril 2021. URL : http://journals.openedition.org/ studifrancesi/35431; DOI : https://doi.org/10.4000/studifrancesi.35431

\section{Ce document a été généré automatiquement le 18 avril 2021.}

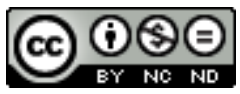

Studi Francesi è distribuita con Licenza Creative Commons Attribuzione - Non commerciale - Non opere derivate 4.0 Internazionale. 


\title{
Aa. Vv., Adverbiaux et topiques
}

\author{
Chiara Preite
}

\section{RÉFÉRENCE}

AA. VV., "Travaux de linguistique française», Adverbiaux et topiques, $\mathrm{n}^{\circ} 47,2003$, pp. 184. Les articles rassemblés dans ce volume portent sur la question, encore floue du point de vue terminologique et conceptuel, de la topicalité ou thématicité des constituants adverbiaux.

1 MICHEL CHAROLLES, De la topicalité des adverbiaux détachés en tête de phrase, pp. 11-49. Les SN référentiels dans les constructions disloquées sont très fréquents à l'oral en français et ils fonctionnent comme des topiques, car le détachement d'un sN suivi de sa reprise sous forme d'un pronom clitique facilite l'intégration des informations. Dans cet article M. Charolles se propose de montrer quel est le bénéfice fonctionnel de ce type de construction. Pour ce faire, l'auteur aborde l'examen de quelques phrases exemplificatives, en partant du point de vue du sujet qui doit produire ou traiter un tel énoncé, ce qui lui permet d'avancer des hypothèses sur les contextes en amont et en aval dans lesquels les adverbiaux détachés en tête de phrase s'avèrent les mieux placés. Ensuite Charolles présente des études sur les liens que les sous-phrases adverbiales antéposées entretiennent avec le discours précédent (Ramsay 1984 et Ford \& Thompson 1986), ainsi que sur les relations entre topique et commentaire (Jacobs 2001), notamment sur les opérations d'adressage et de cadrage. En conclusion, l'auteur soutient que la capacité d'établir un critère pour la répartition des informations textuelles - le potentiel cadratif - en fonction de la spécificité des circonstances attachées à la communication d'une phrase, confère aux adverbiaux cadratifs un rôle proéminent dans la cohésion discursive: ils guident le lecteur dans l'accès à la cohérence du discours parce qu'ils précèdent et évoquent des informations avant leur même énonciation.

SOPHIE PRÉVOST, Les compléments spatiaux: du topique au focus en passant par les cadres, pp. 51-77. 
3 Dans cette étude, S. Prévost considère les rapports que des notions telles que «topique» et «cadre» entretiennent entre elles dans une perspective phrastique. L'auteur soutient que, bien que nettement distincts, les deux concepts doivent être envisagés dans la continuité l'un de l'autre. En effet, le topique et le cadre peuvent être considérés comme les pôles d'un continuum dont la zone intermédiaire montre un partiel recouvrement entre les deux. Après avoir essayé de délimiter les définitions des deux notions, partant des nombreuses définitions proposées par la littérature, l'auteur montre à travers quelques exemples que les topiques détachées et non liés sont assimilables aux cadres qui limitent d'un point de vue notionnel l'applicabilité de la prédication qui suit: cela signifie qu'à l'intérieur de l'axe topique/cadre il existe une zone mixte. Ensuite, Prévost analyse les compléments nominaux spatio-temporels, communément étudiés du côté du cadrage, du point de vue du topique: le circonstant en tête de phrase, du fait de sa nature référentielle, peut assumer un statut de topique, sans abandonner sa fonction de cadrage. Ce sera le contexte qui enlèvera l'ambiguïté interprétative. L'auteur conclut avec l'examen syntactico-sémantique aussi bien que pragmatico-discursif d'un corpus d'occurrences où apparaissent en co-présence une préposition et une reprise nominale, tiré de FRANTEXT et de GOOGLE.

CATHERINE FUCHS et NATHALIE FOURNIER, Du rôle cadratif des compléments localisants initiaux selon la position du sujet nominal, pp. 79-109.

5 Cette contribution porte sur la position du sujet dans une phrase indépendante (sv/Ns) qui présente un terme introducteur initialisé (x) et sur le rapport entre la position du sujet et les opérations constitutives de l'énoncé. Les auteurs s'interrogent sur le rôle cadratif ou non qui peut être revêtu par le terme initialisé $x$, qui fonctionne comme un introducteur du cadre référentiel spatio-temporel validant le discours et qui n'est donc pas autonome. À cette fin, C. Fuchs \& N. Fournier suivent un parcours qui les conduit d'abord à analyser les paramètres co-textuels qui se révèlent solidaires de la position du sujet antéposé ou postposé; ensuite à interpréter les configurations qui en résultent en termes d'opérations sous-jacentes constitutives de l'énoncé; enfin à examiner la dimension discursive des configurations Xsv et xvs dans un contexte inter-énoncé. Il en résulte que ces deux types d'énoncés se caractérisent par des opérations sous-jacentes différentes qui permettent aux auteurs d'établir la dimension cadrative de $\mathrm{x}$ dans XsV et non cadrative dans xvs.

6 KAREN LAHOUSSE, La complexité de la notion de topique et l'inversion du sujet nominal, pp. 111-136.

7 K. Lahousse ouvre sa contribution par un excursus sur la littérature abondante et diverse concernant le concept de topique, et se propose de la raffiner en distinguant plusieurs types d'éléments qui n'ont que quelques traits topicaux communs. En particulier l'auteur s'attache à montrer que pour formuler les conditions dans lesquelles l'inversion du sujet nominal en français peut apparaître de manière légitime, ni une approche qui fait appel à la topicalité d'un élément antéposé ni une approche purement syntaxique ne sont suffisantes: il faut distinguer le «topique scénique» et le «topique contrastif», comme deux éléments partageant certaines caractéristiques du prototype «topique». Le topique scénique d'une proposition correspond à la localisation spatio-temporelle ou abstraite où l'événement dénoté par le verbe a lieu et il explique l'inversion nominale dans les phrases principales non interrogatives où un adverbe ou un groupe prépositionnel se trouve en position initiale, ou bien dans les inversions absolues. En revanche, le topique contrastif est envisagé comme un topique 
prototypique et il correspond à un constituant disloqué qui bloque l'inversion nominale à cause de sa valeur contrastive.

BERNARD COMBETTES, Le topique comme constituant périphérique: aspects diachroniques, pp. 137-161.

Selon B. Combettes les «constituants périphériques» possèdent des caractéristiques syntaxiques et discursives presque autonomes qui semblent leur permettre de revêtir certaines fonctions textuelles spécifiques dans le domaine énonciatif aussi bien que dans celui de la cohérence textuelle. Une étude diachronique de ces éléments périphériques, notamment des topiques, porte sur plusieurs points d'intérêt: au niveau syntaxique, il est possible d'en examiner le degré d'intégration à la structure de la proposition qui peut être l'objet d'une évolution; en revanche, au niveau discursif, l'attention se focalise sur les changements éventuels dans les fonctions pragmatiques véhiculées. Dans cet article, l'auteur tente de montrer que la catégorie des unités topicales introduites par un marqueur a obéi, dans son évolution, aux règles de la grammaticalisation générale: un groupe d'élément s'est constitué en une seule unité dont les composantes ont perdu leurs propriétés syntaxiques et référentielles de départ pour acquérir des valeurs textuelles et énonciatives. En ce qui concerne le topique, Combettes remarque que le passage de la fonction de circonstant d'un élément à celle de cadre, puis à celle de topique s'accompagne d'un fonctionnement discursif influencé par certaines structures textuelles du texte argumentatif qui lui accordent une portée large dépassant le cadre de la phrase. Dans un phase évolutive suivante, le topique désormais mieux intégré aux liens syntaxiques - connaît une restriction de la portée au domaine phrastique. Cette conclusion rejoint l'hypothèse de S. Dik, mentionnée par Combettes, selon laquelle le figement des constructions topicalisées est à l'origine de certaines réalisations du thème.

DOMINIQUE KLINGLER, Spécificité du dispositif créé par le marqueur «wa» en japonais. Comparaison avec le français, pp. 163-179.

11 Cet article traite du fonctionnement de la particule japonaise wa en tant que topique, qui peut être postposée directement à un nom ou à une expression nominale (SN wa) ou bien postposée à un syntagme circonstanciel, lui-même postposé à un nom ( $\mathrm{sN}$ de wa). Dans les deux cas wa détache les éléments auxquels il est postposé du reste de l'énoncé, tout en permettant d'établir un lien sémantique avec la suite de l'énoncé: en effet, wa contribue à délimiter un cadre de validation pour la suite de l'énoncé, en affectant le constituant qui le suit d'un statut de «circonstant cadratif». D. Klingler explique par comparaison avec cette analyse la fonction topicale des adverbiaux détachés en tête de phrase en français. Les constructions en wa trouvent en français leur équivalent dans l'extraposition et l'utilisation d'un pronom tonique: dans les deux langues les circonstants cadratifs ne constituent pas le topique de l'énoncé en tête duquel ils se trouvent, c'est-à-dire ce sur quoi il va porter le discours, mais ils peuvent persister comme cadres pour un paragraphe, voire pour un texte en entier. 